\title{
A Market-Driven Framework Towards Environmentally Sustainable Mobile Computing
}

\author{
Siny Joseph \\ Arts, Sciences, and Business \\ Kansas State University at Salina \\ siny@k-state.edu
}

\author{
Vinod Namboodiri, Vishnu C. Dev \\ Electrical Engineering and Computer Science \\ Wichita State University \\ \{vinod.namboodiri, \\ vxchersusoladev\}@wichita.edu
}

\begin{abstract}
Amid the plethora of initiatives and research endeavors targeting the minimization of power and energy consumption of information and communication technologies (ICT), what has been largely missing is an effort to reduce the energy consumption and electronic waste generated by the rapidly growing segment of mobile computing and communication devices. One "green" approach to meet both the goals of minimizing life cycle energy consumption and reducing electronic waste generation is that of increased device lifespan. Increased device lifespans, however, are possible only if the underlying market forces support such a paradigm shift. This paper develops a market-driven framework for mobile phone devices that helps understand the reasons that affect a firm's decision to offer a green choice for consumers (where "green" is defined as devices with longer lifespan) and considers the feasibility, possible benefits, and challenges in increasing device lifespan.
\end{abstract}

\section{INTRODUCTION}

When it comes to examining energy efficiency and the concept of environmental sustainability in computing, the focus has invariably been on data centers and mobile infrastructures like cell towers, because they have been considered the power hogs within the sector. There has, however, been a big shift in how the general population performs computing and communication tasks these days with a much greater reliance on mobile, battery-operated devices. The increased role of mobile devices has resulted in recent work advocating environmental sustainability in mobile computing (e.g. [1]). The work in [2, 3] found that computing devices, including data centers, server farms, desktops, and mobile devices (laptops and mobile phones), accounted for about $3-7 \%$ of the global electricity usage. Surprisingly, mobile devices were responsible for at least $5-10 \%$ of this share due to their large scale, and this share is expected to grow as power-hungry smart phones proliferate the market [4]. Energy-related research in the fast growing mobile computing area, unfortunately from an environmental perspective, has been focused primarily in addressing only the batterylifetime issue. While optimizing battery lifetime can be useful in reducing the overall energy costs of mobile devices, it only considers energy consumed to operate these devices (i.e, use phase energy), neglecting the energy consumed to

Copyright is held by author/owner(s). manufacture and recycle them. Mobile devices have very short lifespans compared to other computing devices (e.g. desktops), and thus, the overall energy spent (and emissions produced) in manufacturing these devices is a very significant share (upwards of 50\% [5]) of the overall life cycle. The problem of electronic waste is also an important one, with less than $10 \%$ of mobile handsets globally being recycled [5].

One avenue of cutting down energy costs for the non-use phase is greater efficiency in the processes (e.g. manufacturing, transportation, recycling) employed. This avenue is an ongoing approach employed by firms currently to not only cut energy costs, but also overall monetary costs. Another avenue would be increasing the lifespan of mobile devices which would cut down energy consumed for tasks like manufacturing, transportation, and recycling across the entire spectrum of mobile devices. A longer lifespan would mean that the energy consumed by a device during the non-use phases would be amortized over a longer use-phase, in turn reducing the overall energy consumed by the mobile device segment. Furthermore, the increase in lifespan option is expected to have an immediate impact and will be complementary to the ongoing search for efficiencies in processes. It has the added benefit of reducing electronic waste by slowing down the rate of device replacement.

Increase in mobile device lifespan can be achieved only if consumers can be incentivized to retain their devices longer. From a technical perspective, this would require greater emphasis on software features that can be updated, and hardware components that can be replaced to keep up (or stay close) with improved user-experience offered by newer devices. Some recent efforts that have sprung up in this direction include the yet to be released ZTE Eco-Mobius phone, and Project Ara [6], a effort from Google that includes a collaboration with Phonebloks [7]. These efforts are building modular smartphones as "blocks" which can be swapped to reduce the need for replacing the entire device. Additionally, educating consumers on the environmental impact of frequent device replacements may help. However, by and large, an increase in mobile device lifespans will not be easy to achieve relying only on technological advances and educational efforts. The existing mobile device market relies on frequent device turnover to sustain itself and changing that model to a more green one (with greater device lifespans) would require a study of the underlying economics and how it can be used to tune pricing and system design to increase such offerings by firms, and adoption by consumers. ${ }^{1}$. The

\footnotetext{
${ }^{1}$ This extended abstract primarily summarizes the work
} 
objective of this paper is thus to study the underlying market economics of mobile device offerings and understand the competitiveness of longer lifespan (or green) offerings.

The research focus in the direction of green mobile computing in this paper will be on mobile phone devices as they tend to have the highest turnover among mobile devices, possibly induced as a result of frequently introduced software and hardware features, one or two year wireless carrier contracts, and the customer perception of a new "free" phone. As retention of such mobile phone devices also depends on the nature of cellular service received, the proposed economic framework will be applied to a device package as a whole, where a package includes a device (handset) and a cellular service plan.

\section{ECONOMIC FRAMEWORK}

The proposed economic framework that will be developed in this paper will consider the scenario of a firm introducing a green choice (called Firm 1) where consumers sign a longer contract in exchange for a possibly cheaper package (combination of device and service plan), which can be supplemented with software and hardware upgrades/maintenance to improve user-experience as the device ages. By comparing the demand for Firm 1 in a differentiated Bertrand competition (see [8]) with another firm (Firm 2) that offers only a shorter duration contract, but possibly newer, better hardware more frequently, a better understanding of the feasibility and challenges in consumers adopting the green choice (offered by Firm 1) can be gained.

\subsection{Modeling Methodology}

For simplicity and intuitive results, it is assumed that two firms characterize the market (a 2-player market). The mobile industry is concentrated with few major players, so modeling two firms (duopoly) is expected to capture at least some of the market dynamics. Firm 1 offers consumers a mobile device package with a contract of $t_{1}$ years, while Firm 2 offers a package with a contract for $t_{2}$ years, where $t_{1} \geq t_{2}$. If Firm 2 represents the current practice of short duration contracts, then Firm 1 can be thought of as the firm offering a green choice to consumers. Consumer preference is characterized based on the model of vertical product differentiation by Mussa and Rosen in [8]. In a vertically differentiated product space, all consumers agree over the most preferred mix of characteristics and, more generally, over the preference ordering [9]. Vertical differentiation explicitly accounts for differences in consumer attitudes towards mobile phone packages that consist of a hardware communications device and a fixed duration service plan contract, with a device replacement offered after the contract expires.

Let $k_{1}, k_{2}$ be positive real numbers that describe the average user-experience provided by a firm's offered device over the entire contract duration. The user-experience value of a device being offered by the $i$ th firm, $k_{i}$, will take the duration of contracts offered into consideration. For example, a larger duration of $t_{1}$ can be expected to have a smaller value of $k_{1}$ as this requires the consumer to retain an old device longer. The popularity of a particular device brand or manufacturer can be similarly incorporated in the user-experience parameter. $\theta$, again a positive real number, is a taste pa-

from the journal article in [11] and incorporates some additional discussions based on newer developments in the area. rameter. All consumers prefer higher user-experience for a given price; a consumer with high $\theta$ is more willing to pay for higher user-experience. $\theta$ can be modeled as a distribution of tastes in the economy according to some density $f(\theta)$ with a cumulative distribution function $F(\theta)$. In this work a uniform distribution in the interval $[0,1]$ was assumed for the taste parameter $\theta$. Let $p_{1}$ and $p_{2}$ be the prices paid by the consumer for each of the options respectively.

A consumer has the following preferences:

$$
U= \begin{cases}\theta k_{1}-p_{1}+s, & \text { chooses Firm } 1 \text { with longer contract } \\ \theta k_{2}-p_{2}, & \text { chooses Firm } 2 \text { with shorter contract } \\ 0, & \text { chooses no package }\end{cases}
$$

where $k_{1} \leq k_{2}$. U can be thought as the utility derived from the use of the mobile device for an offered subsidy $s$ (described below). The utility is separable in user-experience and price.

The impact of any subsidies governments and NGOs offer is factored into the above model by the term $s$ for the environmental cost ${ }^{2}$ added to Equation 1. This could be a combination of costs incurred for manufacturing and recycling of a device, and dealing with associated carbon emissions. Increasing lifespan results in amortizing this cost over a longer duration and reduces the annual environmental costs. This reduction in annual cost can be passed along as a subsidy/annual cost saving to the consumer to help improve the chances of adoption.

The demand functions for both the shorter contract (Firm 2) and longer contract (Firm 1) options can be subsequently expressed and used to solve a profit maximization problem, from which equilibrium quantities $Q_{1}$ and $Q_{2}$, and prices of packages desired $p_{1}$ and $p_{2}$, can be computed.

\section{EVALUATION RESULTS}

The objective of the evaluations here is to gauge the competitiveness of a firm that offers an option for consumers that requires retaining their mobile phone longer (than another competing firm) and analyze the impact of economic incentives. This is because eventually the introduction of such a sustainable choice will depend on the market share it can gather. The profit maximization problems presented in the previous section were solved using Mathematica. A consumer has three options available in the 2-player model: choose Firm 1 with a longer duration plan, choose Firm 2 with a shorter duration plan, or decide against adopting a package from either firm. The relative user experience ratio $\frac{k_{1}}{k_{2}}$ was set to various values between 0 and 1 in these experiments with $k_{2}$ kept constant. The cost functions were modeled as $c_{i}=0.5 \beta k_{i}^{2}$, for $i=1,2$ where each firms's cost incurred is a function of the user-experience it provides with $\beta$ acting as a scaling parameter ${ }^{3}$ We use the values of $\beta=50 E-06$ and $k_{2}=1000$ in our experiments unless specified otherwise; these values along with $\theta$ (Equation 1), chosen from a uniform distribution $[0,1]$, ensure that the profit and quantity values stay positive and meaningful. With the ratio $\frac{k_{1}}{k_{2}}$ varied from 0 to 1 , a small value of the ratio $\frac{k_{1}}{k_{2}}$

\footnotetext{
${ }^{2}$ This could be a monetary value attached to the emissions and fossil fuels used up. Carbon offset costs in terms of planting new trees could be one way to perceive this term. ${ }^{3}$ This is a commonly used form of modeling costs in economic theory [10].
} 
would imply a vast difference in user-experience making it more difficult for Firm 1 to compete with Firm 2. The point of interest was to look at what ratios Firm 1 is competitive.

With no subsidies offered, $s=0$, Figure 1(a) shows that initially when user-experience of the device from Firm 1 is low, Firm 2 captures about half the market with the remaining split between Firm 1 and the no device package option. As $k_{1}$ increases, Firm 1 captures an increasing share of the market eventually gaining a third of the market, mainly at the expense of the no device package option. Firm 2's market share also increases. A takeaway from this plot is that Firm 1 is reasonably competitive with over a quarter of market share at all times for the parameter values considered $\left(\beta=50 E-06, k_{2}=1000\right)$; however, Firm 2 is always dominant.

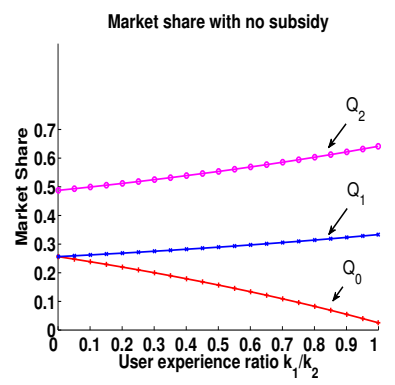

(a)

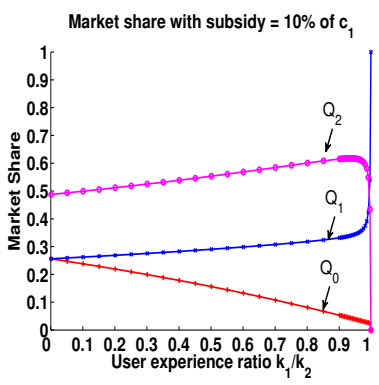

(b)
Figure 1: Market share of a firm offering a green choice without and with subsidy; even a small subsidy makes the firm more competitive.

Next, the impact of subsidies is studied on how much market share the green choice offered by Firm 1 can garner for a 2-player market, and whether the user-experience ratio needed differs significantly from the scenario with no incentives. As Figure 1(b) shows, adding a value of subsidy greatly benefits Firm 1 in terms of the market share it is able to capture from its competitors; in fact when $k_{1}$ is very close to $k_{2}$ Firm 1 is able to capture the entire market. These results indicate that offering subsidies can make a firm offering a green option competitive, if the derived user-experience is comparable.

\section{IMPLICATIONS FOR SYSTEM DESIGN}

Based on the results in the previous section, it is apparent that user-experience on a device, in addition to costs and incentives, plays a major role in determining whether consumers will adopt an older mobile device package over a newer mobile device package. So how can the user-experience on older mobile devices packages be improved? In terms of the analytical model presented earlier, this question asks how the value of parameter $k_{1}$ can be brought closer to $k_{2}$. This could be done through software-based or hardwarebased approaches. In the former approach, software could be written to provide latest features (available on newer hardware) on older hardware. Paradigms such as cloudcomputing could be used to overcome some of the resourceconstraints on older hardware. For example, remote cloud servers could be leveraged for applications that include computation intensive tasks such as image processing as opposed to relying on local hardware. Hardware-based approaches could rely on newer paradigms where mobile devices are constructed as a set of replaceable blocks [6, 7]. Based on the evolving needs of a user, some of these blocks could be replaced with other blocks, balancing user-experience with the need for manufacturing new blocks. Additional details and discussion on how to increase user-experience on older mobile devices can be found in [11].

\section{CONCLUSIONS}

One approach to environmentally sustainable mobile computing is for users to retain their mobile devices longer as a means to reduce life cycle energy costs and electronic waste. This paper studied the conditions under which market economics will support a firm offering a sustainable choice of cellular phone service to consumers considering the possibility of some loss in user-experience. The competitiveness of a firm that offers such sustainable longer duration service plans was studied in comparison to a firm offering shorter contracts, including the impact of subsidies to incentivize the adoption of such plans. Addition of subsidies and any underlying cost advantages by offering a longer-term contract was found to aid competitiveness greatly, with the condition that user-experience over the lifetime of the device is close (not necessarily equal) to the other firm offering a shorter-term contract.

Some additional important questions related to this direction of work addressed through discussions in [11] (but still need further research) are: What is the incentive for device unit manufacturers to have longer lifespans for their devices?, Who should be responsible for providing incentives for the adoption of environmentally-friendly mobile devices, and how would these incentives look like?, and How will the results of this project apply to a wider class of mobile devices?

\section{REFERENCES}

[1] R. Puustinen and G. Zadok, "The Green Switch: Designing for Sustainability in Mobile Computing," in First USENIX International Workshop on Sustainable Information Technology, February 2010.

[2] P. Somavat, S. Jadhav, and V. Namboodiri, "Accounting for the energy consumption of personal computing including portable devices," in Proceedings of the 1st International Conference on Energy-Efficient Computing and Networking, ser. e-Energy '10, 2010, pp. 141-149.

[3] P. Somavat and V. Namboodiri, "Energy consumption of personal computing including portable communication devices," Journal of Green Engineering, vol. 1, no. 4, pp. 447-475, 2011.

[4] M. P. Mills, "The cloud begins with coal. big data, big networks, big infrastructure, and big power. an overview of the electricity used by the digital ecosystem," http://www.tech-pundit.com/wpcontent/uploads/2013/07/Cloud_Begins_With_Coal.pdf?c761ac.

[5] Nokia, "Sustainability Report," 2011. [Online]. Available: http://www.nokia.com/global/about-nokia/people-and-planet/

[6] Google, "Project Ara," www.projectara.com/.

[7] "Phonebloks - A phone worth keeping," https://phonebloks.com/en/goals.

[8] M. Mussa and S. Rosen, "Monopoly and product quality," Journal of Economic Theory, vol. 18, pp. 301-317, 1978.

[9] J. Tirole, The theory of industrial organization. The MIT Press, 1988.

[10] T. L. Saitone and R. J. Sexton, "Impacts of minimum quality standards imposed through marketing orders or related producer organizations," American Journal of Agricultural Economics, vol. 92, no. 1, pp. 164-180, 2010.

[11] S. Joseph, V. Namboodiri, and V. Dev, "Towards environmentally sustainable mobile computing through an economic framework," Emerging Topics in Computing, IEEE Transactions on, vol. PP, no. 99, pp. 1-1, 2014. 